\title{
Reversible Data Hiding Scheme using Prediction Error Expansion in Pixel Value Blocking and Ordering
}

\author{
Satyajit De \\ Department of Computer Science, \\ Maheshtala College, Budge Budge Trunck Road, \\ Kolkata-700141, West Bengal, India.Email: \\ Alok Haldar \\ Department of Computer Science, \\ Kharagpur College, West Midnapur,

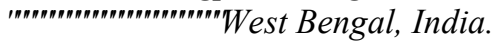

\author{
Biswapati Jana \\ Department of Computer Science, \\ Vidyasagar University, West Midnapur, \\ West Bengal, India.
}

\begin{abstract}
This paper presents a better reversible data hiding method depending on pixel value ordering and prediction-error expansion technique. A host image is first segmented into non-overlapping sub-blocks of adjacent three pixels and ordered them as ascending order. For each sub-block, the maximum pixel value is predicted by the second maximum pixel value. Then the second maximum pixel value is predicted by the minimum pixel value or minimum pixel value is predicted by the second maximum pixel value. Then by using prediction-error expansions, we can insert one or two secret bits into every sub-block pixels and also we can recover the hidden secret bits and restore the cover image fully from watermarked image. Experimental results of this method demonstrate that the embedding capacity and PSNR value is larger than another pixel value ordering and prediction error expansion based approach. Also, the visual quality of the obtained marked image is better than other PVO and PEE based method
\end{abstract}

Keywords: Pixel-value ordering, Reversible data hiding, Prediction-error expansion, Adjacent pixel grouping.

\section{INTRODUCTION}

In the spatial domain different reversible data hiding methods have been divided as lossless compression [3-4], difference expansion (DE) [5-8], histogram shifting (HS) [11-12], prediction- error expansion (PEE) [15], etc. in the spatial domain. DE technique and LSB embedding scheme are used to get a minimum image distortion with high embedding capacity. In adjacent pixels blocks, Alattar applied difference expansion technique to embedded secret bits [7]. To increase the embedding capacity, Al-Qershi et al. used a twodimensional difference expansion technique (2D-DE) with a threshold value depending on the image behavior [8]. The multilevel histogram technique is used by Zhao et al. to embed more secret bits [11]. In his method to enhance embedding capacity secret bits are modulated by using more peak points. Luo et al. generate a strong connection among different pixel blocks to produce a difference histogram and multi-level histogram shifting to embed the secret data [10].
A lossless data hiding method based on a histogram modification is proposed by $\mathrm{Ni}$ et al., where the zero or minimum points of the image histogram are utilized [17].

A reversible data hiding method with prediction-error expansion is introduced by Thodi and Rodriguez.

$\mathrm{Hu}$ et al. introduced a modify reversible data hiding scheme by reducing the overflow location map. Li et al. Proposed an improvement by using adaptive embedding and pixel selection. Lee et al. proposed a reversible data hiding scheme that is free of location map and a corresponding predictive value is derived from the average of its adjacency pixels to make little bit predictive errors.

Li et al. proposed a reversible data hiding scheme [1] using pixel-value-ordering and prediction-error expansion. After sorting in ascending order of every non-overlapped sub-block of equal sizes, the second maximum or minimum pixel value was used to predict the maximum pixel or minimum pixel value respectively. Then by applying prediction-error expansion technique secret data was embedded. Best result was achieved in this technique by using $2 \times 2$ sub-blocks i.e. 4 pixels' sub-block. But for this method, maximum pixels are not used to improve the embedding capacity as well as the image quality for every sub-block and this improvement is done by Jung's method.

Jung's proposed a reversible data hiding scheme [2] using pixel-value-ordering and prediction-error expansion. To improve Li et al's scheme Jung's divide the cover image into three pixels non-overlapped sub-blocks. For each sub-block, sorted the pixels in ascending order and then the second largest pixel value was used to predict the maximum pixel value. Then to embed secret data prediction error expansion was applied into it. As a result, high embedding capacity and good image quality occurs than Li et al's method. But still there is a space available to improve the embedding capacity as well as the image quality for every sub-block and this improvement is done by the proposed method.

In this paper to improve Jung's method a new reversible data hiding scheme is proposed for three pixels' sub-blocks based on pixel value ordering with prediction error expansion. Three pixels of each sub-blocks are ordered in ascending order and 
two prediction errors is calculated. One is between maximum and second maximum pixel value and the other between second maximum and minimum pixel value. Depending on these prediction errors it is tested that the sub-blocks can be embed two/one secret bits. Then by applying pixel-error expansion two/one secret data is hided into the sub-blocks and then all secret bits can be fetched and restore the cover image from watermarked image completely. As a result, embedding capacity and image quality becomes high.

\section{RELATED WORK}

\section{Review of Li et al.'s Scheme}

Li et al.'s used in [1] Pixel value ordered based predictor with Pixel error expansion based reversible data embedding scheme. For secret bits embedding according to the Li et al.'s method [1], the cover image is divided into different nonoverlapped equal-sized sub-blocks. For a chosen block, sort the pixel values in ascending order and then it use the second largest pixel value to predict the maximum pixel or second smallest pixel value to predict minimum pixel. Best performance (For embedding capacity and PSNR value) is achieved in his paper by taking $2 \times 2$ size blocks. Suppose a 2 $\times 2$ block of sorted order pixels with pixel values (si, si+1, $s i+2, s i+3)$. It used the second largest value $s i+2$ to predict the maximum value $s i+3$. For this the prediction error is calculated as e $2=s i+3-s i+2$. Prediction error e 2 is modified to e2' as follows.

$$
\mathrm{e}_{2}^{\prime}= \begin{cases}\mathrm{e}_{2} & \text { if } \mathrm{e}_{2}=0 \\ \mathrm{e}_{2}+\mathrm{p}_{2} & \text { if } \mathrm{e}_{2}=1 \\ \mathrm{e}_{2}+1 & \text { if } \mathrm{e}_{2}>1\end{cases}
$$

Where $\mathrm{p}_{2}$ is a secret bit either 0 or 1 to be embedded.

Now the maximum pixel $s_{i+3}$ is modified to $s_{i+3}{ }_{i+3}$ to store secret bit $\mathrm{p}_{2}$ as follows

Where $\mathrm{p}_{2}$ is a secret bit either 0 or 1 to be embedded.

Now the maximum pixel $s_{i+3}$ is modified to $s^{\prime}{ }_{i+3}$ to store secret bit $\mathrm{p}_{2}$ as follows

$$
s^{\prime} \quad= \begin{cases}s_{i+a} & \text { if } e_{2}=0 \\ s_{i+a}+p_{2} & \text { if } e_{2}=1 \\ s_{i+a}+1 & \text { if } e_{2}>1\end{cases}
$$

It also used the second smallest value $s_{i+1}$ to predict the minimum value $s_{\mathrm{i}}$. For this the prediction error is calculated as $\mathrm{e}_{1}=\mathrm{s}_{\mathrm{i}}-\mathrm{s}_{\mathrm{i}+1}$. Prediction error $\mathrm{e}_{1}$ is modified to $\mathrm{e}_{1}{ }^{\prime}$ as follows.

$$
e_{1}^{\prime}= \begin{cases}e_{1} & \text { if } e_{1}=0 \\ e_{1}-p_{1} & \text { if } e_{1}=-1 \\ e_{1}-1 & \text { if } e_{1}<-1\end{cases}
$$

Where $\mathrm{p}_{1}$ is a secret bit either 0 or 1 to be embedded.
Now the minimum pixel $s_{i}$ is changed to $s_{i}{ }^{\prime}$ to store secret bit $\mathrm{p}_{1}$ as follows

$$
s_{i}^{\prime}= \begin{cases}s_{i} & \text { if } e_{1}=0 \\ s_{i}-p_{1} & \text { if } e_{1}=-1 \\ s_{i}-1 & \text { if } e_{1}<-1\end{cases}
$$

Now, the marked block is obtained as $\left(s^{\prime}{ }_{i}, s_{i+1}, s_{i+2}, s^{\prime}{ }_{i+3}\right)$.

Again for data extraction from maximum pixel the prediction error is calculated as $\mathrm{e}_{2}^{\prime}=\mathrm{s}_{\mathrm{i}+3}^{\prime}-\mathrm{s}_{\mathrm{i}+2}$.

If $\mathrm{e}_{2}=0$ then this sub-block does not contain secret bit for maximum pixel and the original pixel is unchanged. If $1 \leq \mathrm{e}_{2}{ }^{\prime}$ $\leq 2$ then this sub-block contains a secret bit for maximum pixel and the secret bit is $\mathrm{p}_{2}=\mathrm{e}_{2}{ }^{\prime}-1$ and the original maximum pixel is restored as $\mathrm{s}_{\mathrm{i}+3}=\mathrm{s}^{\prime}{ }_{\mathrm{i}+3}-\mathrm{p}_{2}$. If $\mathrm{e}_{2}^{\prime}>2$ then this subblock does not contain any secret bit for maximum pixel and the original maximum pixel is restored as $s_{i+3}=s_{i+3}^{\prime}-1$.

Again for data extraction from minimum pixel the prediction error is calculated as $\mathrm{e}_{1}^{\prime}=\mathrm{s}_{i}^{\prime}-\mathrm{s}_{\mathrm{i}+1}$. If $\mathrm{e}_{1}^{\prime}=0$ then this sub-block does not contain secret bit for minimum pixel and the original pixel is unchanged. If $-2 \leq \mathrm{e}_{1}{ }^{\prime} \leq-1$ then this sub-block contains a secret bit for minimum pixel and the secret bit is $p_{1}$ $=\left|\mathrm{e}_{1}{ }^{\prime}\right|-1$ and the original minimum pixel is restored as $\mathrm{s}_{\mathrm{i}}=\mathrm{s}_{\mathrm{i}}^{\prime}$ $+\mathrm{p}_{1}$. If $\mathrm{e}_{1}{ }^{\prime}<-2$ then this sub-block does not contain any secret bit for minimum pixel and the original minimum pixel is restored as $\mathrm{s}_{\mathrm{i}}=\mathrm{s}_{\mathrm{i}}^{\prime}+1$.

But for this method, still there is a space available to improve the embedding capacity as well as the image quality for every sub-block and this improvement is done by Jung's method [2].

\section{Review of Jung's Scheme}

Jung used [2] PVO based predictor and PEE based reversible data embedding scheme to embed secret bits. In this method cover image is divided into three pixels non-overlapped subblocks. For a chosen sub-block, sort the pixels in ascending order and then using second largest pixel value predict the maximum pixel to embed data like $\mathrm{Li}$ et. al.'s scheme. Suppose a three-pixel sub-block of sorted order with pixel values is $\left(\mathrm{s}_{\mathrm{i}}, \mathrm{s}_{\mathrm{i}+1}, \mathrm{~s}_{\mathrm{i}+2}\right)$.

It used the second largest value $s_{i+1}$ to predict the maximum value $\mathrm{s}_{\mathrm{i}+2}$. For this the prediction error is calculated as $\mathrm{e}=\mathrm{s}_{\mathrm{i}+2}$ $\mathrm{s}_{\mathrm{i}+1}$. Prediction error e is modified to $\mathrm{e}^{\prime}$ by Jung as follows.

$$
e^{\prime}= \begin{cases}e & \text { if } e=0 \\ e+p & \text { if } e=1 \\ e+1 & \text { if } e>1\end{cases}
$$

Where $\mathrm{p}$ is a secret bit either 0 or 1 to be embedded.

Now the maximum pixel $s_{i+2}$ is changed to $s^{\prime}{ }_{i+2}$ to store secret bit $\mathrm{p}$ as follows

$$
s_{i+2}^{\prime}=\left\{\begin{array}{lr}
s_{i+2} & \text { if } e=0 \\
s_{i+2}+p & \text { if } e=1 \\
s_{i+2}+1 & \text { if } e>1
\end{array}\right.
$$


Now, the marked sub-block is obtained as $\left(\mathrm{s}_{\mathrm{i}}, \mathrm{s}_{\mathrm{i}+1}, \mathrm{~s}^{\prime}{ }_{\mathrm{i}+2}\right)$.

Again for data extraction from maximum pixel the prediction error is calculated as $\mathrm{e}^{\prime}=\mathrm{s}^{\prime} \mathrm{i}+2-\mathrm{s}_{\mathrm{i}+1}$.

If $\mathrm{e}^{\prime}=0$ then this sub-block does not contain secret bit for maximum pixel and the original pixel is unchanged.

If $1 \leq \mathrm{e}^{\prime} \leq 2$ then this sub-block contains a secret bit for maximum pixel and the secret bit is $\mathrm{p}=\mathrm{e}^{\prime}-1$ and the original maximum pixel is restored as $s_{i+2}=s^{\prime} i+2-p$.

If $\mathrm{e}^{\prime}>2$ then this sub-block does not contain any secret bit for maximum pixel and original maximum pixel is restored as $s_{i+2}=s_{i+2}^{\prime}-1$. But in Jung's method it is not used that the minimum pixel value to predict the second maximum pixel again to embed secret bits or does not used the second largest pixel value to predict the minimum pixel to embed secret bits, therefore still there is a space available to improve the embedding capacity and image quality of every sub-block. In the previous method besides the overflow or underflow checking no other checking are used to skip the blocks which are not capable to store secret bits, therefore PSNR values becomes low. Now a better reversible data hiding scheme is proposed to improve EC, PSNR and image quality than the $\mathrm{Li}$ et al.'s and Jung's scheme in the following section.

\section{Motivation and Objective}

Motivation and objective of the proposed scheme is to increase embedding capacity using PVO based PEE technique within the cover image without compromising visual quality. Here, the capacity is measured by bpp and image quality is measured by PSNR (dB). The proposed scheme achieve good visual quality maintaining high embedding capacity and compred better result than the existing schemes.

\section{Proposed Scheme}

In the proposed method based on pixel-value ordering and prediction error expansion we establish a better reversible watermarking scheme for the three-pixel non-overlapped sub blocks. The embedding and extracting procedures are as follows.

\section{Embedding Procedure}

\section{Algorithm for data embedding}

The detailed algorithm for embedding secret data is described as follows:

Input: A cover image of order $\mathrm{r} \times \mathrm{c}$ and the sequence of secret bits which have to be embedded to this image.

Output: A watermarked image of order $r \times c$

Step 1: Read a cover image of size $\mathrm{r} \times \mathrm{c}$ from user.

Step 2: This cover image is divided into three pixels nonoverlapping sub-blocks.
Step 3: Consider a generalise $\mathrm{i}^{\text {th }}$ sub-block containing three pixels as $\left(c_{i}, c_{i+1}, c_{i+2}\right)$.

Step 4: Pixels values $\left(c_{i}, c_{i+1}, c_{i+2}\right)$ are sorted in ascending order to obtain $\left(\mathrm{s}_{\mathrm{i}}, \mathrm{s}_{\mathrm{i}+1}, \mathrm{~s}_{\mathrm{i}+2}\right)$ (i.e. $\left.\mathrm{s}_{\mathrm{i}} \leq \mathrm{s}_{\mathrm{i}+1} \leq \mathrm{s}_{\mathrm{i}+2}\right)$.

Step 5: Prediction errors are calculated as follows

$$
\begin{aligned}
& e_{2}=s_{i+2}-s_{i+1} \\
& e_{1}=s_{i+1}-s_{i}
\end{aligned}
$$

Step 6: (Construction of Location map)

[ Here we use an array $\operatorname{lmap}()$ as a location map. $\operatorname{lmap}(i)=0$, indicates that $\mathrm{i}^{\text {th }}$ sub-block does not contain any secret bits. $\operatorname{lmap}(i)=1$ indicates that secret bits exists either at the maximum pixel $\left(\mathrm{s}_{\mathrm{i}+2}\right)$ or at second maximum pixel $\left(\mathrm{s}_{\mathrm{i}+1}\right)$ or both of them in the $i^{\text {th }}$ sub-block and also $\operatorname{lmap}(\mathrm{i})=2$, indicates that secret bits exists either at the maximum pixel $\left(\mathrm{s}_{\mathrm{i}+2}\right)$ or at minimum pixel $\left(\mathrm{s}_{\mathrm{i}}\right)$ or both of them in that sub-block.]

[Skip the sub-block by setting the location map value to 0 i.e. $\operatorname{lmap}(\mathrm{i})=0$, if it is not capable to embed data.]

We check the embedding capability of the $\mathrm{i}^{\text {th }}$ sub-block using the following conditions

$$
\text { i) } \begin{aligned}
& \mathrm{s}_{\mathrm{i}}= 0 \text { or } \mathrm{s}_{\mathrm{i}+2}=255 \text { [Condition for underflow } \\
& \text { or overflow] } \\
& \text { ii) } \mathrm{e}_{2} \neq 1 \text { and } \mathrm{e}_{1} \neq 1
\end{aligned}
$$

If any one of the above conditions hold then this sub-block have no capability to embed data and we skip the sub-block by setting the location map value to 0 i.e. $\operatorname{lmap}(i)=0$. Store the sorted pixels $\left(\mathrm{s}_{\mathrm{i}}, \mathrm{s}_{\mathrm{i}+1}, \mathrm{~s}_{\mathrm{i}+2}\right)$ into the corresponding positions and ultimately we get $\left(c^{\prime}, c_{i+1}^{\prime}, c^{\prime}{ }_{i+2}\right)$. Then go to the next subblock by setting $i=i+1$ and continue from step 3 otherwise go to step 7 .

Step7: Prediction error $\mathrm{e}_{2}$ is modified as follows

$$
e_{2}^{\prime}= \begin{cases}e_{2} & \text { if } e_{2}=0 \\ e_{2}+p_{2} & \text { if } e_{2}=1 \\ e_{2}+1 & \text { if } e_{2}>1\end{cases}
$$

Where $\mathrm{p}_{2}$ is a secret bit data either 0 or 1 has to be embedded.

Step 8: Now the maximum pixel $s_{i+2}$ is changed to $s_{i+2}^{\prime}$ to store a secret bit $\mathrm{p}_{2}$ as follows

$$
s_{i+2}^{\prime}= \begin{cases}s_{i+2} & \text { if } e_{2}=0 \\ s_{i+2}+p_{2} & \text { if } e_{2}=1 \\ s_{i+2}+1 & \text { if } e_{2}>1\end{cases}
$$

Step 9: Now the prediction error $\mathrm{e}_{1}$ is modified as follows

$$
e_{1}^{\prime}= \begin{cases}e_{1} & \text { if } e_{1}=0 \\ e_{1}+p_{1} & \text { if } e_{1}=1 \\ e_{1}+1 & \text { if } e_{1}>1\end{cases}
$$

Where $\mathrm{p}_{1}$ is a secret bit data either 0 or 1 has to be embedded. 
Step 10: If $e_{1}=0$ then skip the pixels $s_{i+1}$ and $s_{i}$ from data embedding and these pixels remains unchanged. Now the resultant watermarked $i^{\text {th }}$ sub-block is $\left(s_{i}, s_{i+1}, s^{\prime}{ }_{i+2}\right)$. Set the location map value to 1 i.e. $\operatorname{lmap}(i)=1$ to indicate that secret bits may exists either in maximum pixel or second maximum pixel or both. Store the pixels $\left(s_{i}, s_{i+1}, s_{i+2}^{\prime}\right)$ into the corresponding positions and ultimately we get $\left(\mathrm{c}^{\prime}{ }_{i}, \mathrm{c}^{\prime}{ }_{\mathrm{i}+1}, \mathrm{c}^{\prime}{ }_{\mathrm{i}+2}\right)$. Then go to next sub-block by setting $\mathrm{i}=\mathrm{i}+1$ and continue from step 3 otherwise go to step 11 .

Step 11: Now the second maximum pixel $s_{i+1}$ is changed to $s^{\prime}{ }_{i+1}$ to store a secret bit $p_{1}$ provided $s^{\prime}{ }_{i+1}<s^{\prime}{ }_{i+2}$. For that we calculate a value $\mathrm{v}$ as follows:

$$
v= \begin{cases}s_{i+1}+p_{1} & \text { if } e_{1}=1 \\ s_{i+1}+1 & \text { if } e_{1}>1\end{cases}
$$

Step 12: If $v<s^{\prime}{ }_{i+2}$ then the value $v$ is effected on the second maximum pixel $s_{i+1}$ and it is changed to $s^{\prime}{ }_{i+1}=v$. Therefore, we get the watermarked $i^{\text {th }}$ sub-block as $\left(s_{i}, s^{\prime}{ }_{i+1}, s^{\prime}{ }_{i+2}\right)$. Set the location map to 1 for this sub-block i.e. $\operatorname{lmap}(i)=1$ to indicate that secret bits may exists either in maximum pixel or second maximum pixel or both.

\section{Otherwise}

The minimum pixel $s_{i}$ is changed to $s_{i}^{\prime}$ to store secret bit $p_{1}$ as follows

$$
s_{i}^{\prime}= \begin{cases}s_{i}-p_{1} & \text { if } e_{1}=1 \\ s_{i}-1 & \text { if } e_{1}>1\end{cases}
$$

Therefore, we get the watermarked $i^{\text {th }}$ sub-block as $\left(s_{i}{ }^{\prime}, s_{i+1}\right.$, $\left.s^{\prime}{ }_{i+2}\right)$. Set the location map to 2 for this sub-block i.e. $\operatorname{lmap}(i)=2$ to indicate that secret bits may exists either in maximum pixel or minimum pixel or both.

Step 13: Store the watermarked $i^{\text {th }}$ sub-block pixels into the corresponding positions and we get $\left(\mathrm{c}^{\prime}{ }_{i}, \mathrm{c}^{\prime}{ }_{\mathrm{i}+1}, \mathrm{c}^{\prime}{ }_{\mathrm{i}+2}\right)$.

Step 14: Go to the next sub-block by setting $i=i+1$ and continue the above procedure from step 3 until all data bits are embedded.

Step 15: Consider the last data embedding sub-block number is bl. Now the array of location map values "lmap()" is losslessly compressed by arithmetic coding and store the compressed location map with auxiliary information (i.e. last sub-block number bl and length of the compressed location map bits $\left.l_{\mathrm{len}}\right)$ by replacing $\mathrm{X}=2 *\left\lceil\left(\log _{2} M\right)\right]+\operatorname{len}_{\mathrm{lm}}[$ where $\mathrm{M}=$ total number of pixels in cover image] least significant bits of image pixels from beginning. Before replacing the least significant bits of $\mathrm{X}$ pixels from beginning we store the least significant bits of $\mathrm{X}$ pixels into remaining blocks starting from the block no $(\mathrm{bl}+1)$ using the same procedure as above. Finally, we get a watermarked image.

\section{Numerical examples for data embedding}

$$
a_{i+1}^{\prime}=\left\{\begin{array}{lll}
a_{i+1}+p_{0} & \text { if } & a_{i+1}+p_{0}<a_{i+2}^{\prime} \\
a_{i+1}-p_{0} & \text { if } & a_{i+1}-p_{0}>a_{i}^{\prime}
\end{array}\right.
$$
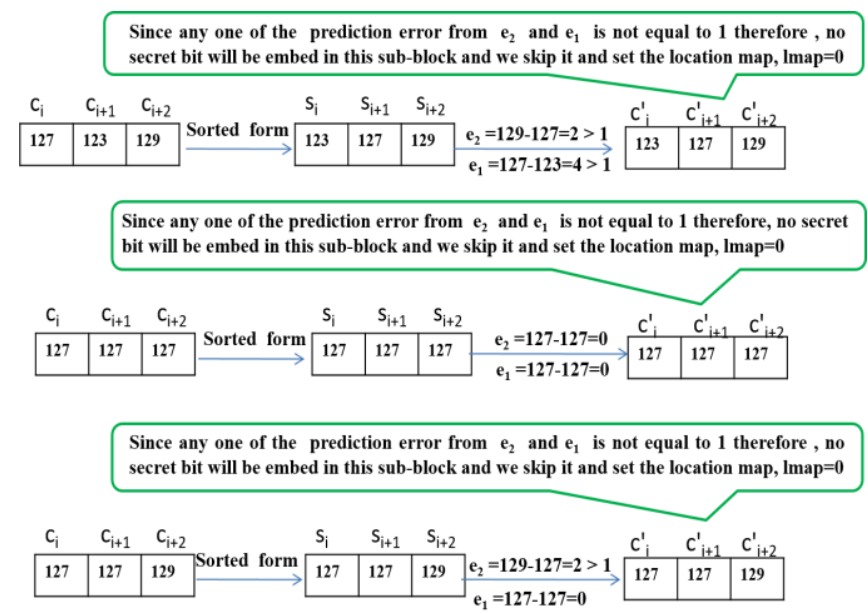

Fig 1: Example of sub-blocks which are not capable to embed secret bits.

In the above examples (i.e. in Fig 2) of sub-blocks since no prediction errors $e_{1}$ or $e_{2}$ have value 1 , therefore these subblocks have no capability to embed secret bits, hence skip these blocks from embedding. Similarly, the sub-blocks in Fig 3 , have no capability to embed data and skip them from embedding.

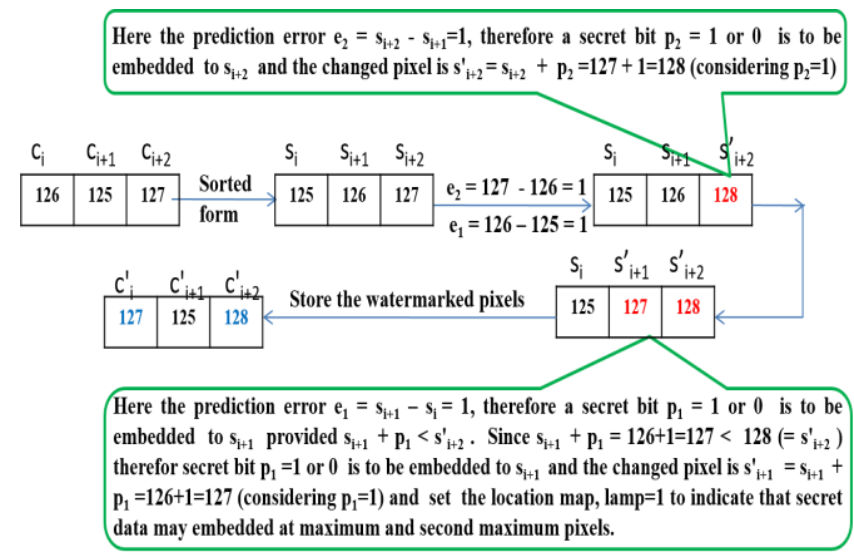

Fig 2: Example of a sub-block which is capable to embed two secret bits into maximum and second maximum pixels with location map value 1 .

In Fig 2, consider a sub-block of pixel values $c_{i}=126, c_{i+1}=$ $125, \mathrm{c}_{\mathrm{i}+2}=127$. After sorting them in ascending order we get $\mathrm{s}_{\mathrm{i}}=125, \mathrm{~s}_{\mathrm{i}+1}=126$ and $\mathrm{s}_{\mathrm{i}+2}=127$. Calculate the prediction errors $\mathrm{e}_{2}=\mathrm{s}_{\mathrm{i}+2}-\mathrm{s}_{\mathrm{i}+1}=127-126=1$ and $\mathrm{e}_{1}=\mathrm{s}_{\mathrm{i}+1}-\mathrm{s}_{\mathrm{i}}=126-125=1$. Suppose we want to store secret bits $\mathrm{p}_{1}=1$ and $\mathrm{p}_{2}=1$. Since $\mathrm{e}_{2}$ $=1$, therefore it is changed to $\mathrm{e}_{2}^{\prime}=\mathrm{e}_{2}+\mathrm{p}_{2}=1+1=2$ and the maximum pixel embed the bit $\mathrm{p}_{2}$ and it is changed to $\mathrm{s}_{\mathrm{i}+2}=\mathrm{s}_{\mathrm{i}+2}$ 
$+\mathrm{p}_{2}=127+1=128$. Similarly, since $\mathrm{e}_{1}=1$ therefore $\mathrm{s}_{\mathrm{i}+1}$ embed the bit $\mathrm{p}_{1}=1$ and $\mathrm{s}_{\mathrm{i}+1}$ is changed to $\mathrm{s}_{\mathrm{i}+1}^{\prime}=\mathrm{s}_{\mathrm{i}+1}+\mathrm{p}_{1}=126+1=127$ $<\mathrm{s}^{\prime}{ }_{\mathrm{i}+2}=128$ and set the location map to 1 . So, we get the watermarked sub-block as $\left(\mathrm{s}_{\mathrm{i}}, \mathrm{s}_{\mathrm{i}+1^{\prime}}, \mathrm{s}_{\mathrm{i}+2^{\prime}}\right)=(125,127,128)$ and they are stored as $\left(\mathrm{c}^{\prime}{ }_{i}, \mathrm{c}^{\prime}{ }_{\mathrm{i}+1}, \mathrm{c}^{\prime}{ }_{\mathrm{i}+2}\right)=(127,125,128)$. We accept $s^{\prime}{ }_{i+1}$ because $s_{i+1}^{\prime}<s_{i+2}^{\prime}$, otherwise we can embed data into $s_{i}$ and set location map to 2 shown in Fig 3. The data extraction for this watermarked sub-blocks are also shown in Fig 4 and

Fig 5 respectively.

Others embedding and extracting situations are as obvious as previous.

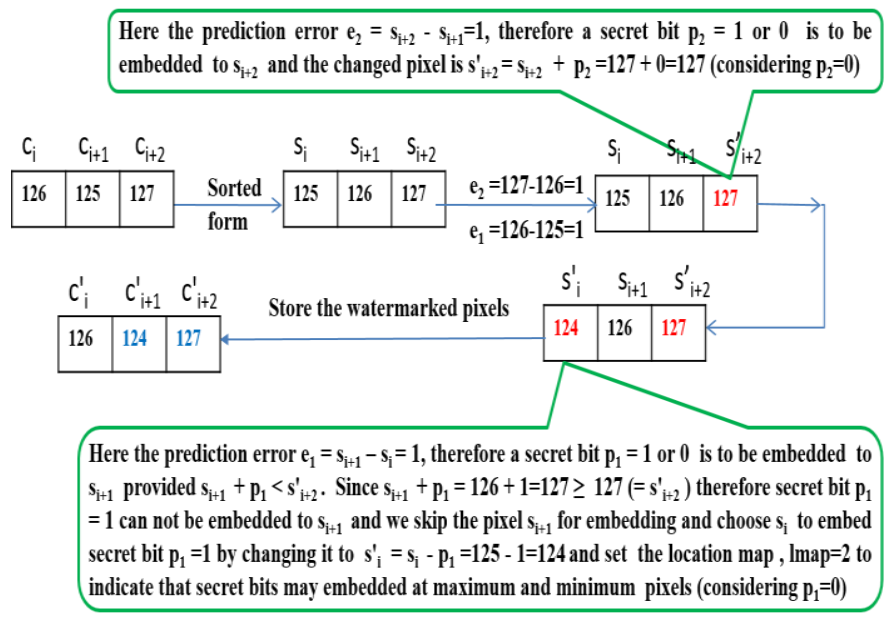

Fig 3: Example of a sub-block which is capable to embed two secret bits into maximum and minimum pixels with location map value is 2

\section{Data Extraction Procedure}

A watermarked image is divided into three pixels nonoverlapping sub-blocks. In Extracting method, we extract the secret bits and restore the pixel values in its original form as follows.

\section{Algorithm for data extraction and image recovery}

Input: A watermarked image of order $\mathrm{r} \times \mathrm{c}$.

Output: Original image i.e. cover image and hided secret bits.

Step 1: To get the auxiliary information [i.e. last embedding block number bl and length of the compressed location map ${ }^{\left.l e n_{l m}\right]}$ from the watermarked image we read first $\mathrm{L}=2 *\left[\left(\log _{2} M\right)\right] \quad$ [where $\mathrm{M}=$ total number of pixels in watermarked image] least significant bits of pixels. Then read next len $\mathrm{n}_{\mathrm{lm}}$ lease significant bits of pixels to get the compressed location map and then decompressing it to get the array of location map values "lamp()".

Step 2: Divide the watermarked image into 3 pixels nonoverlapped sub-blocks starting from pixel number $L+\operatorname{len}_{\mathrm{lm}}+1$. Firstly, by using the following data extraction and image restoration procedure extract the sequence of $\mathrm{X}=\mathrm{L}+\mathrm{le} \mathrm{n}_{\mathrm{Im}}$ hided bits from the sub-block number $(\mathrm{bl}+1)$ to the last block. Now replace the least significant bits of first $\mathrm{X}$ pixels by the sequence of $\mathrm{X}$ extracted bits. Further we apply the following procedure to retrieve the watermark bit sequence and restore the pixels in its original form.

Step 3: $\mathrm{i}=1 \quad / /$ This variable $\mathrm{i}$ indicates the block number.

Step 4: Consider the $\mathrm{i}^{\text {th }}$ sub-block containing pixels as $\left(\mathrm{c}^{\prime} \mathrm{i}\right.$, $\left.\mathrm{c}^{\prime}{ }_{i+1}, \mathrm{c}^{\prime}{ }_{\mathrm{i}+2}\right)$. Now pixels values are sorted in ascending order and we obtain $\left(s_{i,}^{\prime}, s_{i+1}^{\prime}, s_{i+2}^{\prime}\right)$. [i.e. $s_{i}^{\prime} \leq s_{i+1}^{\prime} \leq s_{i+2}^{\prime}$ ].

[ Here the location map $\operatorname{lmap}(\mathrm{i})=0$, indicates that $\mathrm{i}^{\text {th }}$ sub-block does not contain any secret bits. $\operatorname{lmap}(i)=1$ indicates that secret bits may exists either at the maximum pixel $\left(\mathrm{s}^{\prime}{ }_{\mathrm{i}+2}\right)$ or at second maximum pixel $\left(\mathrm{s}^{\prime}{ }_{\mathrm{i}+1}\right)$ or both of them in the $\mathrm{i}^{\text {th }}$ subblock and also $\operatorname{lmap}(\mathrm{i})=2$, indicates that secret bits may exists either at the maximum pixel $\left(\mathrm{s}^{\prime}{ }_{\mathrm{i}+2}\right)$ or at minimum pixel $\left(\mathrm{s}_{\mathrm{i}}^{\prime}\right)$ or both of them in that sub-block.]

Step 5: if $\operatorname{lmap}(i)=0$ then no secret bits exists and pixel values are unchanged. So, store the pixel values of $i^{\text {th }}$ sub-block i.e. $\left(\mathrm{s}_{\mathrm{i},}^{\prime}, \mathrm{s}_{\mathrm{i}+1}^{\prime}, \mathrm{s}_{\mathrm{i}+2}^{\prime}\right)$ to their original positions to get the cover image

sub-block as $\left(c_{i}, c_{i+1}, c_{i+2}\right)$. Then go to the next sub-block by setting $\mathrm{i}=\mathrm{i}+1$ and continue from step 4 otherwise go to next step 6. [end of if]

Step 6: If $\operatorname{lmap}(i)=1$ then

a) [ extract the $2^{\text {nd }}$ secret bit $\mathrm{p}_{1}$ for the $\mathrm{i}^{\text {th }}$ sub-block as follows]

Calculate the prediction error $\mathrm{e}_{1}=\mathrm{s}_{\mathrm{i}+1}-\mathrm{s}_{\mathrm{i}}$, then the secret bit extraction and image restoration for the pixel $\mathrm{s}_{\mathrm{i}+1}^{\prime}$ is as follows.

$>$ If $\mathrm{e}_{1}^{\prime}>2$ then no secret bit exists in $\mathrm{s}_{\mathrm{i}+1}^{\prime}$ and the recovered cover pixel value is $s_{i+1}=s_{i+1}^{\prime}-1$

$>$ If $1 \leq \mathrm{e}_{1} \leq 2$ then the recovered secret bit is $\mathrm{p}_{1}=$ $\mathrm{e}_{1}-1$ and the recovered cover pixel value is $\mathrm{s}_{\mathrm{i}+1}$ $=\mathrm{s}_{\mathrm{i}+1}-\mathrm{p}_{1}$.

$>$ If $\mathrm{e}_{1}=0$ no secret bits are there and $\mathrm{s}_{\mathrm{i}+1}$ becomes unchanged and the cover pixel value is $s_{i+1}=s_{i+1}^{\prime}$

b) [extract the $1^{\text {st }}$ secret bit $\mathrm{p}_{2}$ for the $i^{\text {th }}$ sub-block as follows]

Calculate the prediction error $e_{2}^{\prime}=s^{\prime}{ }_{i+2}-s^{\prime}{ }_{i+1}$, then the secret bit extraction and image restoration for the pixel $\mathrm{s}_{\mathrm{i}+2}$ as follows.

$>$ If $e_{2}^{\prime}=0$, no secret bits exist in $s_{i+2}^{\prime}$ and $s_{i+2}^{\prime}$ becomes unchanged and the cover pixel value is $\mathrm{S}_{\mathrm{i}+2}=\mathrm{S}_{\mathrm{i}+2}^{\prime}$

$>$ If $1 \leq \mathrm{e}_{2}{ }^{\prime} \leq 2$, secret bit is there and the secret bit is $\mathrm{p}_{2}=\mathrm{e}_{2}^{\prime}-1$ and the recovered cover pixel value is $\mathrm{s}_{\mathrm{i}+2}=\mathrm{s}_{\mathrm{i}+2}^{\prime}-\mathrm{p}_{2}$

$>$ If $\mathrm{e}_{2}^{\prime}>2$, no secret bit is there and sub-block is shifted in data embedding and its recovered cover pixel value is $\mathrm{S}_{\mathrm{i}+2}=\mathrm{s}_{\mathrm{i}+2}^{\prime}-1$

So, the required fetched bit sequence is $\mathrm{p}_{2} \mathrm{p}_{1}$ (if they exists) and the recovered cover pixels of $i^{\text {th }}$ sub-block is $\left(s_{i,}^{\prime}, s_{i+1}, s_{i+2}\right)$. Now go to step 8 .

[end of outer if of step 6] 
Step 7: If $\operatorname{lmap}(i)=2$ then

a) [extract $1^{\text {st }}$ secret bit $\mathrm{p}_{2}$ for the $\mathrm{i}^{\text {th }}$ sub-block as follows]

Calculate the prediction error $\mathrm{e}_{2}^{\prime}=\mathrm{s}^{\prime}{ }_{\mathrm{i}+2}-\mathrm{s}_{\mathrm{i}+1}^{\prime}$, then the secret bit extraction and image restoration for the pixel $\mathrm{s}_{\mathrm{i}+2}$ as follows.

$>$ If $\mathrm{e}_{2}{ }^{\prime}=0$, no secret bits exist in $\mathrm{s}^{\prime}{ }_{\mathrm{i}+2}$ and $\mathrm{s}^{\prime}{ }_{\mathrm{i}+2}$ becomes unchanged and the cover pixel value is $\mathrm{s}_{\mathrm{i}+2}=\mathrm{s}_{\mathrm{i}+2}^{\prime}$

$>$ If $1 \leq \mathrm{e}_{2}{ }^{\prime} \leq 2$, secret bit is there and the secret bit is $\mathrm{p}_{2}=\mathrm{e}_{2}^{\prime}-1$ and the recovered cover pixel value is $\mathrm{s}_{\mathrm{i}+2}=\mathrm{s}_{\mathrm{i}+2}^{\prime}-\mathrm{p}_{2}$

$>$ If $\mathrm{e}_{2}^{\prime}>2$, no secret bit is there and subblock is shifted in data embedding and its recovered cover pixel value is $\mathrm{s}_{\mathrm{i}+2}=\mathrm{s}_{\mathrm{i}+2}^{\prime}-1$

c) [ extract the $2^{\text {nd }}$ secret bit $\mathrm{p}_{1}$ for the $\mathrm{i}^{\text {th }}$ sub-block as follows]

Calculate the prediction error $\mathrm{e}_{1}^{\prime}=\mathrm{s}_{\mathrm{i}+1}-\mathrm{s}_{\mathrm{i}}^{\prime}$, then the secret bit extraction and image restoration for the pixel $\mathrm{s}_{\mathrm{i}}$ as follows.

$>$ If $\mathrm{e}_{1}^{\prime}=0$, no secret bits are there and $\mathrm{s}_{\mathrm{i}}$ becomes unchanged and the cover pixel value is $\mathrm{s}_{\mathrm{i}}=\mathrm{s}_{\mathrm{i}}^{\prime}$

$>$ If $1 \leq \mathrm{e}_{1}{ }^{\prime} \leq 2$, secret bit is there and the secret bit is $\mathrm{p}_{1}=\mathrm{e}_{1}^{\prime}-1$ and the recovered cover pixel value is $\mathrm{s}_{\mathrm{i}}=\mathrm{s}_{\mathrm{i}}^{\prime}+\mathrm{p}_{1}$

$>$ If $\mathrm{e}_{1}^{\prime}>2$, no secret bit is there and subblock is shifted in data embedding and its recovered cover pixel value is $\mathrm{s}_{\mathrm{i}}=\mathrm{s}_{\mathrm{i}}^{\prime}+1$

So, the required fetched bit sequence is $\mathrm{p}_{2} \mathrm{p}_{1}$ (if they exists) and the recovered cover pixels of $i^{\text {th }}$ sub-block is $\left(s_{i,}, s_{i+1}^{\prime}, s_{i+2}\right)$.

[end of outer if of step 7]

Step 8: Store the recovered cover pixels of $i^{\text {th }}$ sub-block into its original positions $\left(c_{i}, c_{i+1}, c_{i+2}\right)$. Set $i=i+1$ and the above process continued until all sub-blocks are completed.

Step 9: Ultimately we fetched all hided secret bits from the watermarked image and the cover image restored completely.

\section{Numerical examples of data extraction}

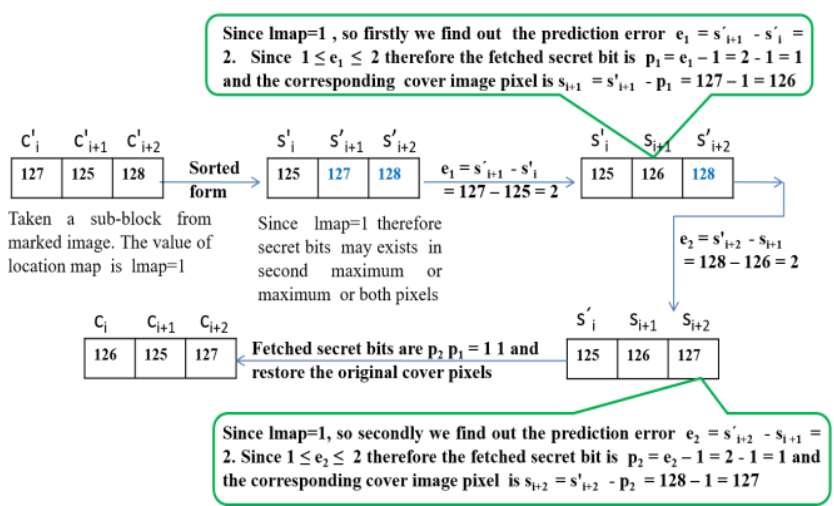

Fig 4: Example of watermarked sub-blocks whose location map value is 1 and two secret bits are fetched from second maximum and maximum pixels depending on prediction error and restore the cover pixels for that sub-block.
In the above fig 4 a watermarked sub-block is considering for data extraction and image recovery. The pixel values of this sub-block are $c^{\prime}{ }_{i}=127, c^{\prime}{ }_{i+1}=125, c^{\prime}{ }_{i+2}=128$ and location map value is 1 i.e. lmap $=1$. After sorting these pixels in ascending order we get $s_{i}^{\prime}=125, s_{i+1}^{\prime}=127$ and $s_{i+2}^{\prime}=128$. Since location map value is 1 , therefore minimum pixel $s^{\prime}{ }_{i}$ does not contain any secret bit but secret bits may exist at maximum $\left(\mathrm{s}^{\prime}{ }_{\mathrm{i}+2}\right)$ and second maximum $\left(s^{\prime}{ }_{i+1}\right)$ pixels. First we have to fetched secret bit (if exists) from second maximum pixel and recovered the pixel value in its original form. Then by using this recovered value of second maximum pixel we have to fetched the secret bit (if exists) from maximum pixel and recovered the pixel it in its original form. The required bit sequence is fetched bit from maximum pixel followed by fetched bit from second maximum pixel. Now calculate the prediction error $\mathrm{e}_{1}=\mathrm{s}^{\prime}{ }_{\mathrm{i}+1}-$ $\mathrm{s}_{\mathrm{i}}^{\prime}=127-125=2$. Since $\mathrm{e}_{1}=2\left(1 \leq \mathrm{e}_{1} \leq 2\right)$ therefore the fetched secret bit is $p_{1}=e_{1}-1=2-1=1$ and the recovered cover pixel is $\mathrm{s}_{\mathrm{i}+1}=\mathrm{s}^{\prime}{ }_{\mathrm{i}+1}-\mathrm{p}_{1}=127-1=126$. Again calculate the prediction error $e_{2}=s^{\prime}{ }_{i+2}-s_{i+1}=128-126=2$. Since $e_{2}=2$ therefore the fetched secret bit $\mathrm{p}_{2}=\mathrm{e}_{2}-1=2-1=1$ and the recovered cover pixel is $s_{i+2}=s^{\prime}{ }_{i+2}-p_{2}=128-1=127$. So, the required fetched bit sequence is $\mathrm{p}_{2} \mathrm{p}_{1}=11$ and the cover pixel sub-block as $\left(\mathrm{s}_{\mathrm{i}}^{\prime}, \mathrm{s}_{\mathrm{i}+1}, \mathrm{~s}_{\mathrm{i}+2}\right)=(125,126,127)$ and they are stored into their original positon as $\left(c_{i}, c_{i+1}, c_{i+2}\right)=(126,125,127)$.

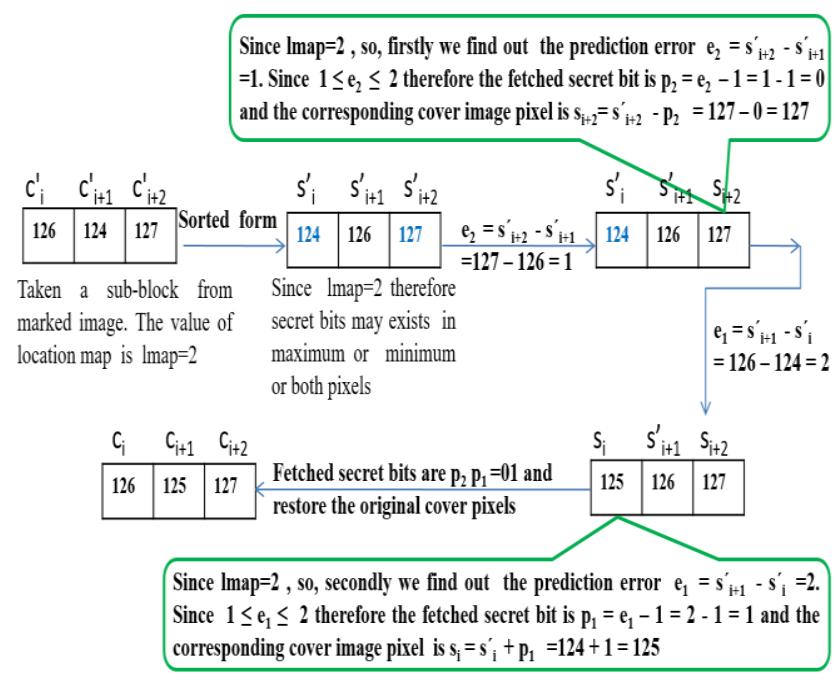

Fig 5: Example of watermarked sub-blocks whose location map value is 2 and two secret bits are fetched from minimum and maximum pixels depending on prediction error and restore the cover pixels for that sub-block.

\section{Experimental Results and Comparisons}

The proposed method is implemented by using MATLAB. The experiment is done by using $512 \times 512$ grey scale cover images taking from USC-SIPT image database and researchget.net. The secret bits are randomly generated in MATLAB using the function $\operatorname{randi}([0,1], 1,1)$. 
Images are used in the proposed scheme as follows.
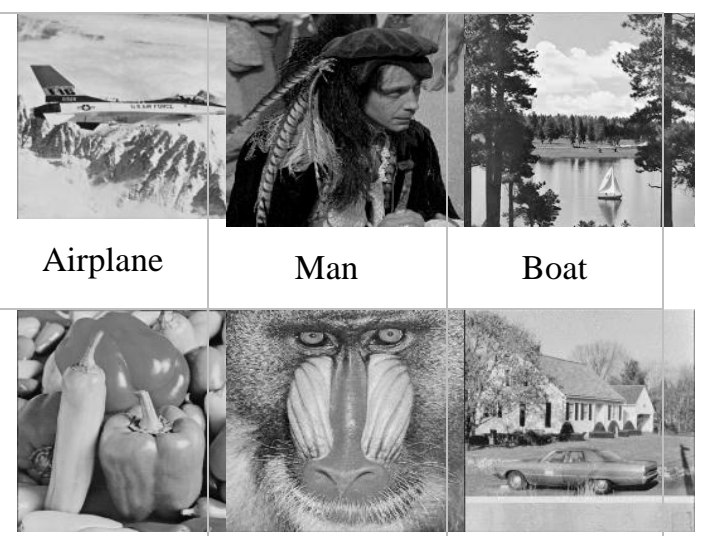

Peppers Baboon House

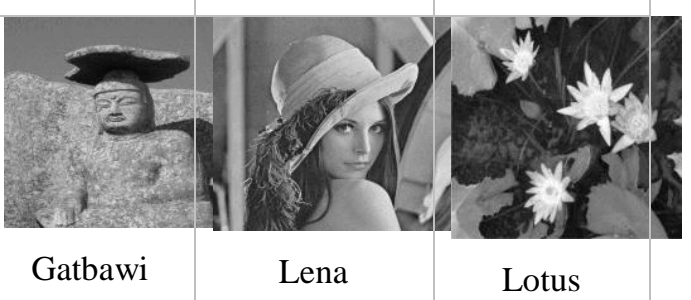

Fig 6: All are gray scale images of size $512 \times 512$ taken from USC-SIPI image database and researchget.net and from google for experiment.

The embedding rate (ER), Peak signal to noise ratio (PSNR) and universal image quality index $\mathrm{Q}$ are generated from the formulas represented in [2] to evaluate the watermarked image quality. In the proposed method each 3 pixels' subblock could contain maximum two bits. Therefore, the total number of secret bits could be embedded in a cover image of order $\mathrm{r} \times \mathrm{c}$ is $(\mathrm{r} \times \mathrm{c}) \times \frac{2}{3}$. So for a $512 \times 512$ grey scale image, maximum secret bits could be embedded in the proposed scheme is $(512 \times 512) \times \frac{2}{3}=174762$ bits.

In the proposed scheme if we neglect a few sub-blocks that contains auxiliary information and location map, then for each other 3-pixels sub-blocks maximum two pixels will be modified to embed secret bits and the other pixels remains unchanged.

Therefore, in the proposed scheme the embedding capacity is more high than the Jung's method and the Li et al.'s method.

Also theoretically the PSNR of the marked image versus its cover image is at least $10 \log _{10} \frac{255^{2}}{M S E} \geq 10 \log _{10} \frac{255^{2} \times a}{2}=$ $49.89 \mathrm{~dB}$.

But practically in the proposed scheme since we skipped all the 3-pixels sub-blocks which have no capability to embed at least one secret bit (i.e. we avoid unnecessary expansions of pixels) therefore the average PSNR value is higher than Jung's method and $\mathrm{Li}$ et al.'s method. Also the average quality index of different images is higher than the Jung's method and Li et al.'s method.

\section{Experimental results of proposed scheme with existing scheme}

The results of proposed scheme compared with $\mathrm{Li}$ et al.'s method [1] and Jung's method [2] using nine standards $512 \times 512$ sized grey scale images are shown in Table 1 and Table 2 as follows.

Table 1: Comparisons of experimental results (Embedding capacity and embedding rate) of proposed method with other method [1,2]

\begin{tabular}{|c|c|c|c|c|c|c|}
\hline & \multicolumn{2}{|c|}{$\begin{array}{c}\text { Li et al.'s method } \\
{[1]}\end{array}$} & \multicolumn{2}{c|}{$\begin{array}{c}\text { Jung's method } \\
{[2]}\end{array}$} & \multicolumn{2}{c|}{$\begin{array}{c}\text { Proposed } \\
\text { method }\end{array}$} \\
\hline $\begin{array}{c}\text { Cover } \\
\text { Image }\end{array}$ & EC & ER & EC & ER & EC & ER \\
\hline Airplane & 12260 & 0.047 & 16098 & 0.061 & 45417 & 0.1733 \\
\hline Man & 8275 & 0.032 & 9533 & 0.036 & 23651 & 0.0902 \\
\hline Boat & 7043 & 0.027 & 8450 & 0.032 & 32415 & 0.1237 \\
\hline Lena & 9799 & 0.037 & 11048 & 0.042 & 38813 & 0.1481 \\
\hline Peppers & 8918 & 0.034 & 10805 & 0.041 & 31763 & 0.1212 \\
\hline Baboon & 3839 & 0.015 & 4508 & 0.017 & 11839 & 0.0452 \\
\hline House & 9910 & 0.038 & 13194 & 0.050 & 32824 & 0.1252 \\
\hline Lotus & 11423 & 0.044 & 12685 & 0.048 & 44775 & 0.1708 \\
\hline Gatbawi & 7105 & 0.027 & 9361 & 0.036 & 31948 & 0.1219 \\
\hline Average & 8730 & 0.033 & 10631 & 0.040 & 32605 & 0.1244 \\
\hline
\end{tabular}

Table 2: Comparisons of experimental results (PSNR and Quality Index) of proposed method with other method [1,2]

\begin{tabular}{|c|c|c|c|c|c|c|}
\hline & \multicolumn{2}{|c|}{$\begin{array}{c}\text { Li et al.'s method } \\
{[1]}\end{array}$} & \multicolumn{2}{c|}{$\begin{array}{c}\text { Jung's method } \\
{[2]}\end{array}$} & \multicolumn{2}{c|}{$\begin{array}{c}\text { Proposed } \\
\text { method }\end{array}$} \\
\hline $\begin{array}{c}\text { Cover } \\
\text { Image }\end{array}$ & PSNR & Q & PSNR & Q & PSNR & Q \\
\hline Airplane & 55.82 & 0.9889 & 56.85 & 0.9910 & 57.38 & 1.0000 \\
\hline Man & 45.32 & 0.9965 & 45.40 & 0.9971 & 58.60 & 1.0000 \\
\hline Boat & 55.91 & 0.9976 & 57.05 & 0.9983 & 58.30 & 1.0000 \\
\hline Lena & 55.56 & 0.9955 & 57.09 & 0.9968 & 57.03 & 0.9999 \\
\hline Peppers & 55.66 & 0.9965 & 57.30 & 0.9976 & 57.37 & 1.0000 \\
\hline Baboon & 56.04 & 0.9995 & 56.97 & 0.9996 & 61.02 & 1.0000 \\
\hline House & 55.95 & 0.9852 & 57.07 & 0.9887 & 58.59 & 1.0000 \\
\hline Lotus & 41.98 & 0.9935 & 42.58 & 0.9949 & 58.03 & 1.0000 \\
\hline Gatbawi & 34.24 & 0.9888 & 31.84 & 0.9909 & 59.23 & 1.0000 \\
\hline Average & 50.72 & 0.9936 & 51.31 & 0.9950 & 58.39 & 0.9999 \\
\hline
\end{tabular}

EC: Embedded capacity, ER: Embedded rate, PSNR: Peak signal to noise ratio, Q: Image quality index

On the experiment, compressed location map bits with auxiliary information are not stored into the watermarked image. On average the embedding capacity of $\mathrm{Li}$ et al.'s method is 8730 bits and Jung's method is 10631 bits, but in the proposed method it is 32605 . This shows that proposed method hides 21974 bits more on average than Jung's method and 23875 bits more than Li et al.'s method. Also the average 
value of ER is 0.0841 more than Jung's method and 0.091 more than $\mathrm{Li}$ et al.'s method.

On the other hand, average PSNR of Li et al.'s method is $50.72 \mathrm{~dB}$ and that of Jung's method is $51.31 \mathrm{~dB}$, but in the proposed method it is $58.39 \mathrm{~dB}$. So, the proposed method improves the average PSNR $7.08 \mathrm{~dB}$ more than Jung's method and $7.67 \mathrm{~dB}$ more than Li et al.'s method. PSNR of all images in the proposed scheme are better than Li et al.'s method and Jung's method except Lena image. The PSNR value of Lena image for proposed scheme is $1.47 \mathrm{~dB}$ more than Li et al.'s method but .06 dB less than Jung's method.

From the above table it is also shown that the average quality index Q is better than the Li et al.'s and Jung's method.

\section{Comparison Graphs}

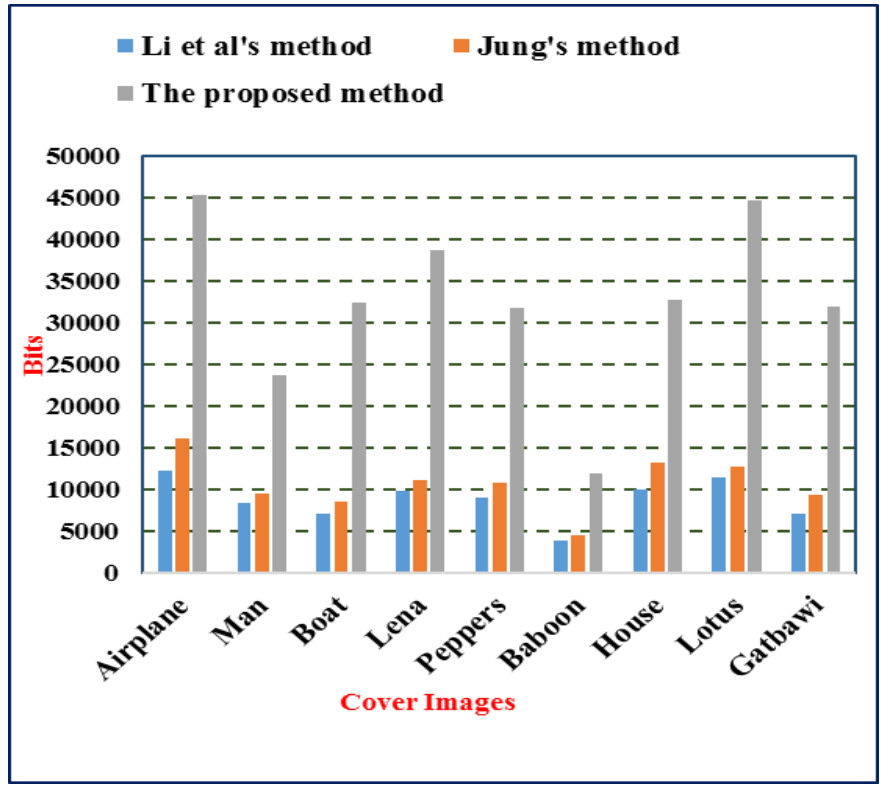

Fig 7: Graph to show a comparison of embedding bits of proposed scheme with existing scheme

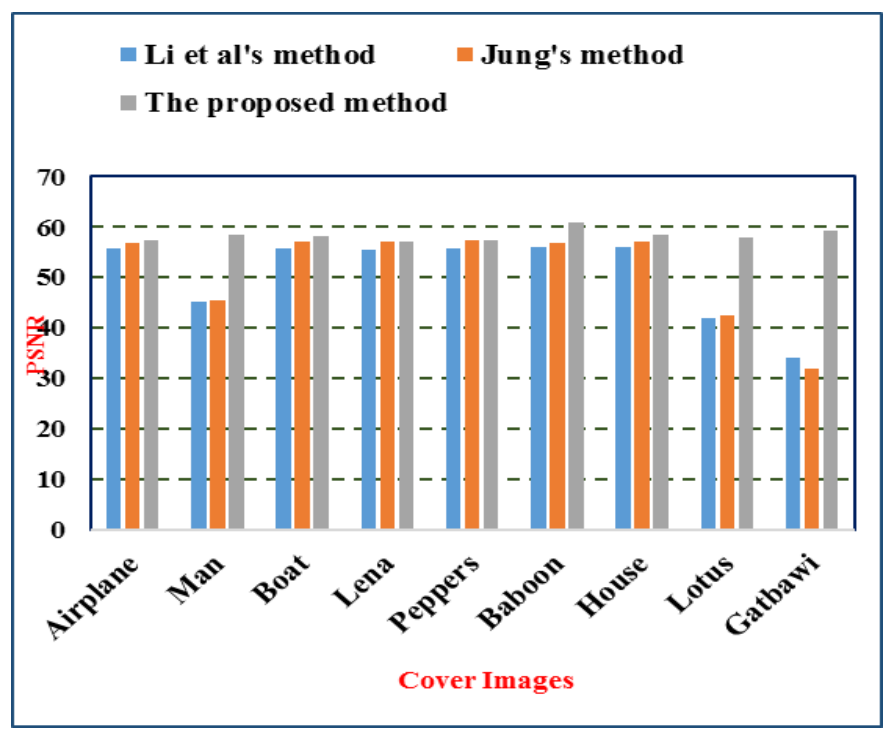

Fig 8: Graph to show a comparison of PSNR proposed scheme with existing schemes

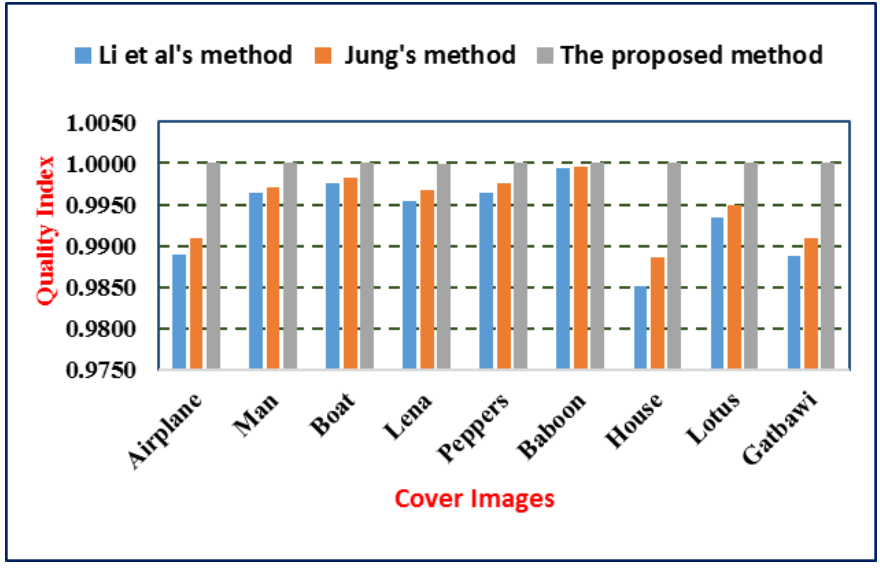

Fig 9: Graph to show a comparison of visual quality index between proposed schemes with existing schemes.

\section{CONCLUSION}

The paper presented a new Pixel-value ordering and Prediction error expansion based reversible watermarking scheme to improve Li et al.'s method and Jung's method. In this scheme, the prediction errors on ordered pixels of three pixels sub-blocks calculated and then skip those blocks which are not capable to embed data. Two secret bits are embeded each remaining sub-block depending on Prediction error expansion technique and finally from the watermarked image we can be fetched the secret bits and restore the cover image completely. Experimental results of the proposed scheme show that the embedding capacity, PSNR, and the quality index is better than Li et al.'s method and Jung's method. In our future work, we try to embed three secret bits into three values pixel blocks to improve embedding capacity as well as keeping good visual quality in different domain using PVO based PEE technique.

\section{REFERENCES}

[1] Li, X., Li, J., Li, B. and Yang, B. Hsiao, J.Y. Highfidelity reversible data hiding scheme based on pixelvalue-ordering and prediction-error expansion. Signal Processing 93(2013) 198-205.

[2] Jung, K.H. Data Hiding Scheme with Reversibility Based on Neighboring Pixel Value Grouping and Ordering. Jour of Adv Research in Dynamical \& Control Systems 10 (2)(2018)227-233.

[3] J. Fridrich, M. Goljan, R. Du, Lossless data embedding - new paradigm in digital watermarking, EURASIP Journal on Applied Signal Processing 2002 (2) (2002) 185-196.

[4] M.U. Celik, G. Sharma, A.M. Tekalp, E. Saber, Lossless generalized- LSB data embedding, IEEE Transactions on Image Processing 14 (2) (2005) 253 266.

[5] Tai, W.L., Yeh, C.M. and Chang, C.C. Reversible data hiding based on histogram modification of pixel 
International Journal of Applied Engineering Research ISSN 0973-4562 Volume 14, Number 8 (2019) pp. 2029-2037

(C) Research India Publications. https://dx.doi.org/10.37622/IJAER/14.8.2019.2029-2037

differences. IEEE Transactions on Circuits and Systems for Video Technology 19 (2009) 906-910.

[6] Tian, J. Reversible data embedding using a difference expansion. IEEE Transactions on Circuits and Systems for Video Technology 13 (2003) 890-896.

[7] Alattar, A.M. Reversible watermark using the difference expansion of a generalized integer transform. IEEE Transactions on Image Processing $\mathbf{1 3}$ (2004) 1147-1156.

[8] Al-Qershi, O.M. and Khoo, B.E. Two-dimensional difference expansion (2D-DE) scheme with a characteristics-based threshold. Signal Processing 93 (1) (2013) 154-162.

[9] F. Peng, X. Li, B. Yang, Adaptive reversible data hiding scheme based on integer transform, Signal Processing 92 (1) (2012) 54-62.

[10] Luo, H., Yu, F.X., Chen, H., Huang, Z.L., Li, H. and Wang, P.H. Reversible datahiding based on block median preservation. Information Sciences 181 (2011) 308-328.

[11] Zhao, Z., Luo, H., Lu, Z.M. and Pan, J.S. Reversible data hiding based on multilevel histogram modification and sequential recovery. AEU -International Journal of Electronics and Communications 65 (2011) 814-826.

[12] Huang, L.C., Tseng, L.Y. and Hwang, M.S. A reversible data hiding method by histogram shifting in high quality medical images. The Journal of Systems and Software 86 (2013) 716-727

[13] M. Liu, H.S. Seah, C. Zhu, W. Lin, F. Tian, Reducing location map in prediction-based difference expansion for reversible image data embedding, Signal Processing 92 (3) (2012) 819-828.

[14] Y. Hu, H.K. Lee, J. Li, DE-based reversible data hiding with improved overflow location map, IEEE Transactions on Circuits and Systems for Video Technology 19 (2) (2009) 250-260.

[15] W. Hong, T.S. Chen, C.W. Shiu, Reversible data hiding for high quality images using modification of prediction errors, Journal of Systems and Software 82 (11) (2009) 1833-1842.

[16] C.C. Lin, N.L. Hsueh, A lossless data hiding scheme based on three- pixel block differences, Pattern hiding based on histogram modification of pixel differences, IEEE Transactions on Circuits and Systems for Video Technology 19 (6) (2009) 906-910. Recognition 41 (4) (2008) 1415-1425.

[17] W.L. Tai, C.M. Yeh, C.C. Chang, Reversible data [18]

P. Tsai, Y.C. Hu, H.L. Yeh, Reversible image hiding scheme using predictive coding and histogram shifting, Signal Processing 89 (6) (2009) 1129-1143. 